\title{
Colorado Medical Students' Attitudes and Beliefs About Marijuana
}

\author{
Michael H. Chan, MD ${ }^{1,2}$, Christopher E. Knoepke, PhD, MSW', Madeline L. Cole, MD ${ }^{1,3}$, \\ James McKinnon, $\mathrm{MA}^{7}$, and Daniel D. Matlock, $M D, M P H^{7}$ \\ 'University of Colorado School of Medicine, Aurora, CO, USA; ${ }^{2}$ University of Texas Health Sciences Center at Houston, Houston, TX, USA; \\ ${ }^{3}$ MetroHealth Medical Center, Case Western Reserve University, Cleveland, OH, USA.
}

BACKGROUND: Over the past two decades, state and local governments across the U.S. have been increasingly reforming marijuana laws. Despite growing support for marijuana as a medical treatment, little is known about medical students' perceptions of marijuana use.

OBJECTIVE: To assess Colorado medical students' personal and professional opinions on current and future marijuana use in a healthcare setting.

DESIGN: A voluntary, anonymous, online cross-sectional survey.

PARTICIPANTS: Medical students $(n=624)$ at the University of Colorado School of Medicine between January and February 2014 were invited to participate.

MAIN MEASURES: Numerical responses were quantified using counts and percentages, and Likert scale responses were collapsed for bivariate analysis. Items were gathered thematically and additively scored for each subscale. Internal consistency reliability statistics were calculated for each subscale to ensure that items were assessing similar constructs. Unadjusted $t$ tests and one-way analysis of variance (ANOVA) were used to calculate mean differences in subscale scores between subgroups.

KEY RESULTS: We received 236 responses (37\%). Students indicated support for marijuana legalization (64\%), and few believed that physicians should be penalized for recommending marijuana to patients (6\%). Nearly all (97\%) believed that further marijuana research should be conducted, and believed marijuana could play a role in the treatment of various medical conditions. Seventy-seven percent reported that they believed marijuana use had the potential for psychological harm, and 68\% indicated concern for potential physical harm. Only a minority of students would recommend marijuana to a patient under current law (29\%), or if it were legally available (45\%). Acceptability of marijuana for treatment of approved conditions was not correlated with age or gender, but was positively correlated with living in Colorado prior to medical school $(p<0.001)$ and with prior marijuana use $(p<0.001)$. CONCLUSIONS: Medical students support marijuana legal reform, medicinal uses of marijuana, and increased research, but have concerns regarding risks of marijuana

Electronic supplementary material The online version of this article (doi:10.1007/s11606-016-3957-y) contains supplementary material, which is available to authorized users.

Received February 23, 2016

Revised July 14, 2016

Accepted December 2, 2016

Published online January 17, 2017 use, and appear hesitant to recommend marijuana to patients.

KEY WORDS: medical culture; medical education; substance abuse; doctor-patient relationships; psychometrics.

J Gen Intern Med 32(4):458-63

DOI: $10.1007 / \mathrm{s} 11606-016-3957-y$

(C) Society of General Internal Medicine 2016

\section{INTRODUCTION}

Colorado has long been at the forefront of marijuana legal reforms, including the creation of a medical marijuana (MMJ) program for approved conditions. MMJ became available in Colorado following passage of Amendment 20 in 2000, becoming an expansive clinical industry over the intervening years. Reports from 2014 estimated that MMJ had been "recommended" (the formal process requiring the approval of a physician) to over 278,000 patients through the state's registry, with 117,239 persons contemporaneously possessing a valid "red card" - approximately $2 \%$ of the state's total population. ${ }^{2}$ Colorado's additional legal reforms, including a 2006 state constitutional amendment decriminalizing marijuana, full legalization for recreational use by adults in $2012{ }^{3}$ and opening of marijuana retail establishments on January $1,2014,{ }^{4}$ have not substantially altered the size or scope of the MMJ industry. However, these measures were passed by way of a democratic process, rather than rulemaking along the lines of medical necessity (e.g., Food and Drug Administration [FDA] approval).

Prior studies have looked at the opinion of physiciansalthough not of medical students - on the topic of MMJ, with diverse findings. A 2005 national study of physicians found physician favorability to be evenly divided on the matter. ${ }^{5} \mathrm{~A}$ 1990 survey of oncologists reported that $33 \%$ of respondents indicated that marijuana should be accepted as medical treatment, and that $53 \%$ of those expressing an opinion believed marijuana should be available by prescription. ${ }^{6}$ More recently, a 2013 study of Colorado family physicians showed that only $19 \%$ of respondents believed that physicians should recommend MMJ. ${ }^{7}$

University of Colorado medical students, by virtue of attending the only allopathic medical school in Colorado, can provide unique insight into training and practicing in a state with widespread availability, use, and political acceptability of 
marijuana. This study presents the results of a survey of University of Colorado medical students about their personal and professional opinions on current and future marijuana use in a healthcare setting. We hypothesize that Colorado medical students will have a tolerant attitude toward marijuana use in healthcare settings.

\section{METHODS}

\section{Study Design}

A cross-sectional survey exploring attitudes and beliefs related to MMJ was distributed to all medical students at the University of Colorado School of Medicine (Aurora, CO).

\section{Participants/Recruitment/Data Collection}

The survey was distributed via email to 624 medical students beginning in January 2014 via Google Forms. Reminders were both sent via university email and placed throughout campus buildings and shared on class-wide online forums. The survey was open for 45 days. No incentive or compensation was provided for participation.

\section{Survey Development}

Items were chosen based on literature review and experiences with $\mathrm{MMJ}$ in educational and clinical settings in the following domains: opinions on legal reform, opinions on medical usefulness of MMJ, beliefs in risk and/or benefits associated with MMJ, support for MMJ training and research, and opinions about consequences for physicians who recommend MMJ for their patients (Online Appendix). Several items were created by adapting those appearing in previously published studies by addressing the trainee's perspective rather than that of a practicing provider. ${ }^{6,7}$ Questions that were not based on previously used questions were developed de novo by the team, which consisted of medical students and faculty. Care was taken to adhere to pre-established principles of survey question design in terms of question format, structure, grouping, and sequence. ${ }^{8}$ Questions were reviewed and revised for clarity and relevance numerous times by the team to ensure that participants understood them and that responses were capturing what the investigators intended. The majority of novel questions were developed by medical students to ensure that content and vocabulary was appropriate to the medical student experience. The survey went through multiple iterations prior to distribution.

Each item asked participants to rate their agreement with various statements on a six-point Likert scale, with responses ranging from "strongly agree" to "strongly disagree" (or "very unacceptable" to "very acceptable" for questions regarding the acceptability of MMJ in medical treatment). Additional questions were asked regarding the sources from which they had learned about marijuana, including several free-response sections. All survey participants were asked demographic questions, including age, sex, prior personal marijuana use, and future career plans.

The study design, protocol, and survey instrument were granted exempt status by the Colorado Multiple Institutional Review Board prior to administration (COMIRB Protocol 132387). No external funding was received for the study.

\section{Data Analysis}

Responses were downloaded from the survey software into Microsoft Excel (2011), and initial numerical analyses were performed using this software. Numerical responses were quantified and summarized using counts and percentages. To allow for bivariate comparisons, we collapsed Likert scale item responses into "agree" (somewhat agree, agree and strongly agree) and "disagree" (somewhat disagree, disagree and strongly disagree). Additional statistical analyses were performed using SPSS software (version 23; IBM Corp., Armonk, NY). Items were grouped according to six constructs: 1) acceptability of MMJ for treatment of approved conditions in Colorado, 2) belief that marijuana use has medical benefits, 3) belief that marijuana use has medical risks, 4) support for marijuana legal reform, 5) support for MMJ training and research, and 6) support for adverse consequences for physicians who recommend MMJ for their patients. Internal consistency reliability statistics were calculated for each subscale to ensure that items were assessing similar constructs. Scores on these constructs were calculated and analyzed for differences according to respondent age, gender, reported history of marijuana use, and whether they had lived in Colorado prior to medical school. To evaluate the mean differences in subscale survey scores between subgroups, independent samples $t$ tests or one-way analysis of variance (ANOVA; both including the Bonferroni correction for multiple comparisons) were conducted where appropriate. Statistical analyses were performed in an exploratory manner without a specific or explicit a priori hypothesis.

\section{RESULTS}

\section{Demographics}

A total of 236 responses were received, for a response rate of $37 \%$. Survey respondents' demographic information is summarized in Table 1. The demographic profile of our sample was generally similar to that of the entire student body, as reflected in archival data provided by the institution. There were no clear differences in response rate by gender $(52 \%$ vs. $48 \%$ male survey respondents vs. institutional enrollment, respectively). Since age was assessed categorically in our survey (to preserve confidentiality), it is not possible to definitively determine whether our sample and the general student population differed by age, but our sample may have been slightly younger than the student population as a whole. The average age for a student during that time frame (across years 
Table 1 Demographics of Survey Respondents

\begin{tabular}{|c|c|c|c|}
\hline \multicolumn{4}{|c|}{ Demographics of Survey Respondents } \\
\hline \multirow[t]{5}{*}{ Age, years } & & Number & Percentage \\
\hline & $\leq 24$ & 65 & $27.7 \%$ \\
\hline & $\overline{2} 5-29$ & 124 & $52.8 \%$ \\
\hline & $30-34$ & 33 & $14.0 \%$ \\
\hline & $\geq 35$ & 13 & $5.5 \%$ \\
\hline \multirow[t]{3}{*}{ Gender } & Female & 109 & $46.6 \%$ \\
\hline & Male & 113 & $48.3 \%$ \\
\hline & Other/prefer not to respond & 12 & $5.1 \%$ \\
\hline \multicolumn{4}{|c|}{ Current year of medical school } \\
\hline & First year & 61 & $25.8 \%$ \\
\hline & Second year & 73 & $30.9 \%$ \\
\hline & Third year & 56 & $23.7 \%$ \\
\hline & Fourth Year & 40 & $16.9 \%$ \\
\hline & Did not respond & 6 & $2.5 \%$ \\
\hline \multicolumn{4}{|c|}{ Educational level } \\
\hline & Bachelor's degree & 214 & $91.5 \%$ \\
\hline & $\begin{array}{l}\text { Master's degree } \\
\text { (including MPH) }\end{array}$ & 32 & $13.7 \%$ \\
\hline & Doctoral degree & 5 & $2.1 \%$ \\
\hline
\end{tabular}

and accounting for different class sizes) was 30 years, suggesting that the average student at the school was older than approximately $80 \%$ of our respondents at the time the study was conducted.

\section{Student Beliefs About Medical Aspects of Marijuana}

Students generally believed that marijuana could play a role in the treatment of the indications approved in the Colorado MMJ program as of 2016 (Fig. 1). When asked specifically, only a minority of respondents expressed belief that marijuana possessed significant benefits to physical health (49\%) and mental health (37\%), and a majority expressed concern that marijuana could cause physical and mental harm (68\% and $77 \%$, respectively). A majority also indicated a belief that marijuana could be addictive (88\%; Table 2).

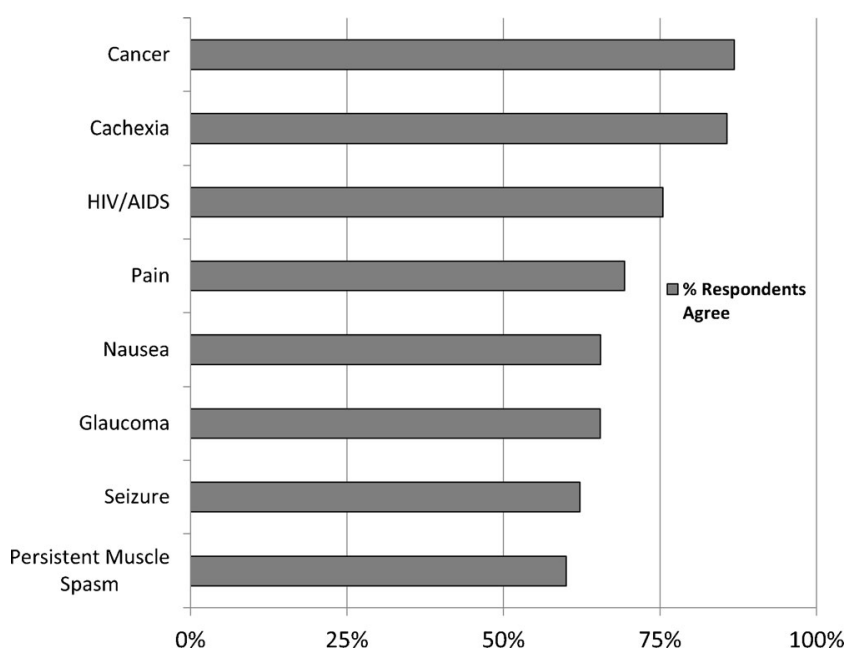

Figure 1 Indications for which medical students believe marijuana can play in a role in medical treatment. These are the eight approved conditions for which physicians can formally recommend marijuana in the State of Colorado.
The overall acceptability of MMJ for treatment of medical conditions did not differ according to age group or gender. Acceptability of MMJ for treatment was related to whether students had lived in Colorado prior to medical school $(t(212)=2.73, p<0.001)$, with those who had not lived in Colorado $(n=59, \mathrm{M}=35.8 / 48)$ scoring MMJ as marginally more acceptable than students who lived in Colorado before starting school ( $n=155, \mathrm{M}=32.1 / 48)$. However, students who reported any previous marijuana use $(n=127, M=35.8 / 48)$ believed MMJ to be more acceptable than those who had never used marijuana ( $n=84, \mathrm{M}=28.8 / 48 ; p<0.001)$. General levels of belief that marijuana holds medical benefits and risk did not differ according to students' gender, age, or whether they had lived in Colorado prior to medical school. When compared to students who had reported never using marijuana, students who had used marijuana reported a greater belief in medical benefits $(n=137, \mathrm{M}=17.44 / 30$ to $n=90, \mathrm{M}=14.38 / 30 ; p<0.001)$ and a lower belief in medical risks associated with marijuana use $(\mathrm{M}=12.56 / 18$ to $\mathrm{M}=13.81 / 18 ; p<0.001$; Table 3$)$.

\section{Student Opinions on Marijuana Policy}

Of the students surveyed, $64 \%$ expressed the belief that marijuana should be legalized for recreational use, with $72 \%$ supporting reclassification of marijuana so that it is no longer a Schedule I substance. Broad support for marijuana legal reform was variable (ranging from 3-18 on a scale of 18; Table 2), and was not associated with age, gender, or having lived in Colorado prior to school. Support for legal changes was related to whether the person had used marijuana $(n=138, M=13.9)$ compared to those who had not used marijuana ( $n=91, \mathrm{M}=10.8$; Table 3 ).

Students also strongly supported eliminating legal/criminal penalties or disciplinary action against the medical licenses of physicians who recommend marijuana to patients $(94 \%$ and $88 \%$, respectively; Table 2). Differences in support for these adverse consequences for physicians also did not differ according to gender, age, or whether students lived in Colorado prior to medical school, but did according to whether students had used marijuana in the past ( $n=137, \mathrm{M}=3.68 / 12$ for those who had; $n=92, \mathrm{M}=4.89$ for those who had not; Table 3). However, only a minority would recommend marijuana to a patient under current law (29\%), or if it were legally available (45\%; Table 2).

\section{DISCUSSION}

The role of MMJ in the treatment of patients with a wide spectrum of medical conditions will continue to be redefined as the social, political, and legal environments evolve. Over $55 \%$ of the United States population lives in a state with reformed marijuana laws. ${ }^{2}$ In Colorado, there were approximately 25 times as many individuals on the Medical Marijuana Registry $^{1}$ as there were persons living with HIV/ $\operatorname{AIDS}^{9}$ at the time this survey was conducted. To the best of our knowledge, ours is the first study to address medical student attitudes and beliefs about marijuana, and the first to 
Table 2 Properties of Six Subscales Related to Marijuana Beliefs of Medical Students

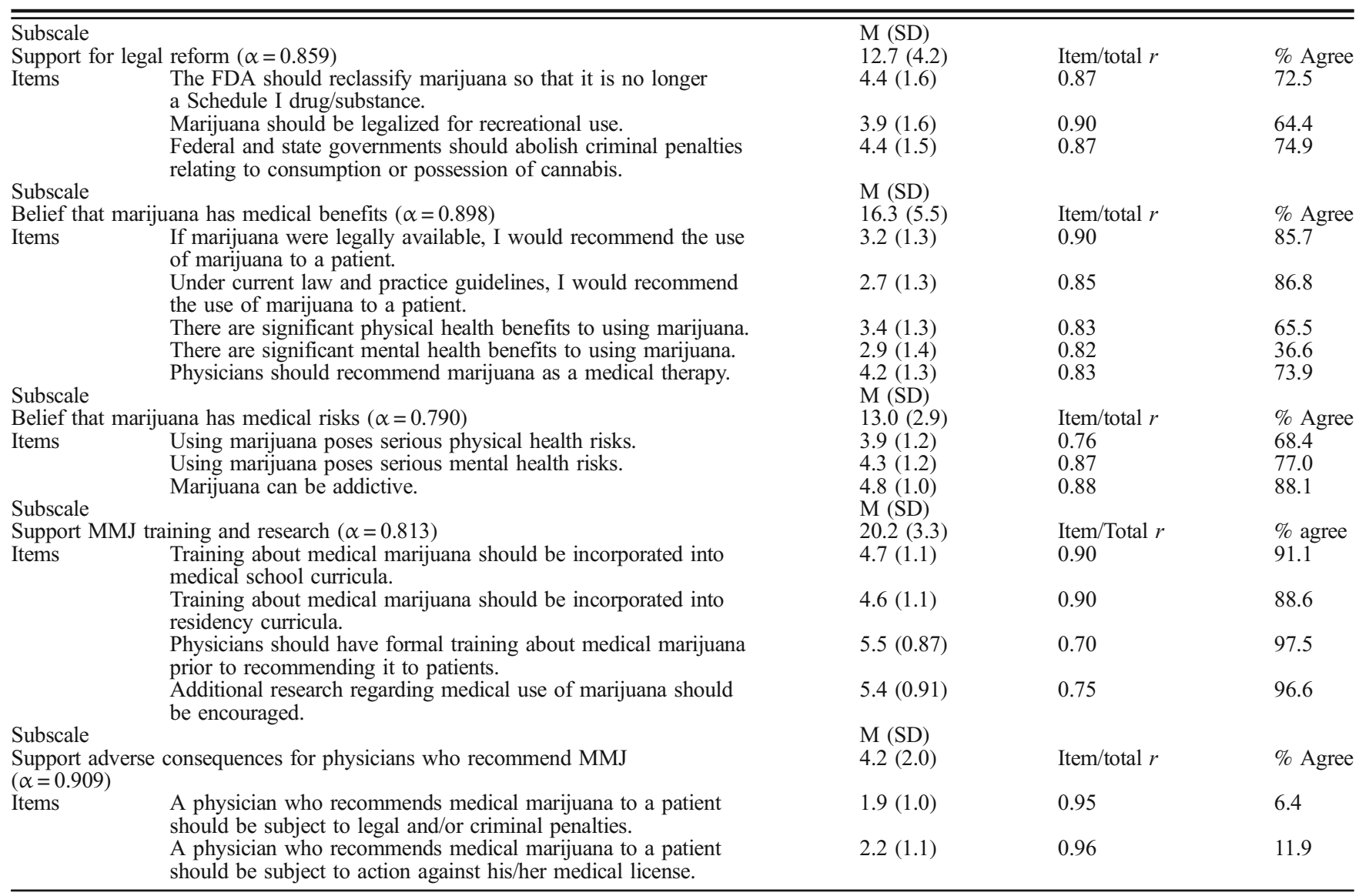

be reported following passage of legal recreational marijuana in Colorado.

\section{Student Attitudes and Beliefs}

Overall, Colorado medical students had strikingly positive attitudes and beliefs toward marijuana. They viewed the legalization of marijuana, medicinal or otherwise, in a favorable manner, and defended the rights of the physicians to recommend marijuana to patients. Students generally felt that MMJ was acceptable in the treatment of indications approved by the Colorado Medical Marijuana Registry, and nearly half felt that marijuana had significant health benefits. In contrast, pain and cancer were the only indication for which a majority of Colorado family physicians had recommended marijuana, and only $27 \%$ of Colorado family physicians felt it had significant physical health benefits. ${ }^{7}$ Student attitudes are more similar to those expressed in a 1991 survey of the American Society of Clinical Oncologists (ASCO), which showed that $48 \%$ of respondents would prescribe marijuana if it were legally available. ${ }^{6}$

However, students were nearly unanimous in calling for further research into the medical usefulness of marijuana, a stance shared with the American College of Physicians, the American Medical Association's Medical Student
Section, and the Institute of Medicine. ${ }^{10-12}$ Improving physicians' and students' knowledge will require a stronger evidence base, as well as increased training opportunities at the undergraduate, graduate, and continuing medical education levels. ${ }^{7}$ Despite strong support for marijuana legal reform, students expressed hesitancy to recommend it themselves, which suggests that medical students may not believe that there is enough data to safely recommend its use to patients and/or may not feel sufficiently trained to prescribe it.

It is possible that non-medical reasons factor into medical students' favorable views of marijuana legal reform. Groups and individuals favor marijuana legal reform for many reasons, including costs related to prohibition enforcement, legal proceedings, and incarceration, as well the economic and noneconomic impact that these have on individuals, families, and society. Previously published studies of physicians have suggested that characteristics including being less religious and political liberalism are associated with greater support for legalizing marijuana, ${ }^{5}$ and support for legalization was associated with "non-moralism" and "permissiveness." 13 Future research should explore whether medical students favor medical legal reform for non-medical reasons, while simultaneously having medically based apprehension for recommending it to patients. 


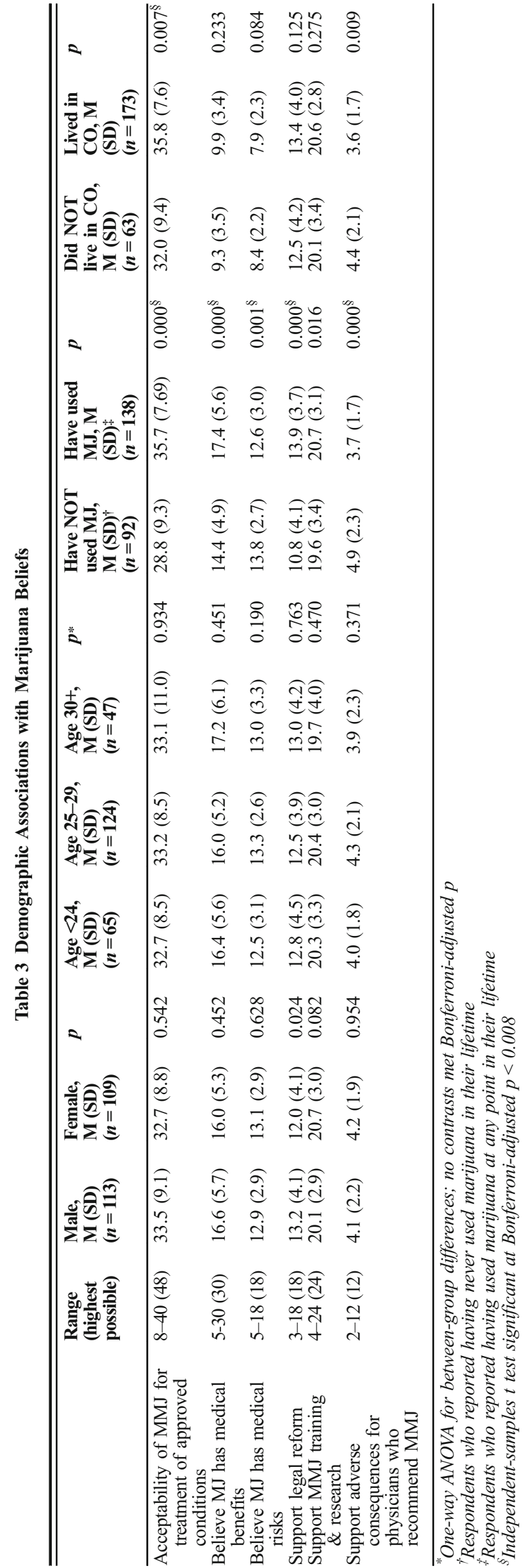

The findings related to prior personal experience with marijuana use are consistent with prior studies showing that individuals with a history of substance use, including marijuana, perceive a lower risk of adverse effects related to use than those who have not. ${ }^{14}$ Hypothesized explanations for these findings include individuals' desire to reconcile past behavior with their perceived identity, perceiving one's own risks with unrealistic optimism, ${ }^{15-17}$ or using one's own lack of negative repercussions to support a belief that risks associated with marijuana use have been overstated historically. It is possible that medical students employ these strategies when assessing the risks and benefits of medical marijuana. These results are also consistent with a previous study of physicians which showed that prior drug use was associated with a favorable opinion of marijuana legalization. ${ }^{13}$

\section{LIMITATIONS}

The primary limitation of our study is our response rate of $38 \%$. However, medical students are a relatively homogeneous group, ${ }^{18}$ and prior studies have shown relatively low response rates in surveys of medical professionals. ${ }^{19}$ We are reassured by the similarity of our reported marijuana use to that of a study published in JAMA ${ }^{20}$ describing drug use in American medical students, and our response rate is similar to those of the ASCO and Colorado Academy of Family Physicians studies that were used in our study's survey development. ${ }^{6,7}$ Demographic comparison between responders and non-responders suggests that our respondents were similar in gender but may have been slightly younger than the overall student body, though statistical comparison is limited. Additionally, subgroup analyses were performed in an exploratory fashion and could not be adjusted for confounding factors. However, these results are consistent with prior studies on physician attitudes with regard to marijuana use. ${ }^{13}$ While our survey had strong face validity and internal reliability, future studies will be necessary to further assess survey validity and to determine which factors are most important in measuring beliefs regarding marijuana among medical students. The single-site design may imply that findings are primarily applicable to University of Colorado students. However, this survey captured a unique moment in the years following the legalization of both medical and recreational marijuana, and as such represents the first group of medical students and professionals encountering marijuana use of this scale in a medical setting.

\section{FUTURE DIRECTIONS}

As more states begin liberalizing marijuana laws, medical schools in other states will experience challenges similar to those seen in Colorado. Since physicians are not the 
only providers confronted by patient marijuana use, surveying other health professionals would have additional merit; however, at the time that this study was completed, physicians were the only level of healthcare providers in Colorado authorized to give patients formal medical marijuana recommendations through the state registry.

\section{CONCLUSIONS}

The topic of marijuana brings up numerous social, cultural, legal, and medical concerns in a way that few other topics do, and much of this seems to occur outside of a medical context. Large numbers of Americans believe that marijuana has medicinal benefit, and many have taken steps to incorporate marijuana into their medical care, regardless of legal consequences. As the general trend toward legalization of marijuana continues, it is the responsibility of the medical field to understand the implications that this substance may have on the people and populations affected. Our study is the first to demonstrate the opinions of medical students on the topic of marijuana, helping to predict future physician attitudes surrounding this culturally charged topic.

Acknowledgements: The authors would like to thank the students of the University of Colorado School of Medicine, the University of Colorado Health Sciences Library, and the University of Colorado Anschutz Medical Campus Office of Student Life for their assistance in conducting this project.

Corresponding Author: Daniel D. Matlock, MD, MPH; University of Colorado School of Medicine, 13199 E. Montview Blvd., Suite 210; Mail Stop F443, Aurora, CO 80045, USA (e-mail: daniel. matlock@ucdenver.edu).

\section{Compliance with Ethical Standards:}

Conflict of Interest: The authors declare that they do not have a conflict of interest. No external funding was used for this project.

\section{REFERENCES}

1. Medical Marijuana Registry Program Update (as of October 31, 2014). Colorado Dept. of Revenue, editor. Denver, Colorado: State of Colorado Department of Public Health \& Environment; 2014.

2. Annual Estimates of the Resident Population for the United States, Regions, States, and Puerto Rico: April 1, 2010 to July 1, 2013. United States Census Bureau, editor. 2013.

3. Breathes W. The history of cannabis in Colorado...or how the state went to pot. Westword. 2012 November 1; 2012.

4. Healy $\mathbf{J}$. Up Early and in Line for a Marijuana Milestone in Colorado. The New York Times. 2014 January 1; 2014.

5. Charuvastra A, Friedmann PD, Stein MD. Physician attitudes regarding the prescription of medical marijuana. J Addict Dis. 2005;24(3):87-93.

6. Doblin R, Kleiman M. Marijuana as antiemetic medicine: a survey of oncologists' experiences and attitudes. J Clin Oncol. 1991;9(7).

7. Kondrad E, Reid A. Colorado family physicians' attitudes toward medical marijuana. J Am Board Fam Med. 2013;26(1):52-60.

8. Dillman DA. Mail and internet surveys: the tailored design method. New York: Wiley; 2000.

9. Integrated Epidemiological Profile of HIV and AIDS Prevention and Care Planning reported through December 2010. Denver, CO: State of Colorado: 2010.

10. Taylor T. Supporting research into the therapeutic role of marijuana. American College of Physicians: 2008 Position Paper [Internet]; 2008:1-20 pp.

11. American Medical Association - Medical Student Section Digest of Policy Actions, 2008. 2012.

12. Kollas CD. Medical marijuana: what should palliative care specialists know? J Pain Symptom Manag. 2012;13(2):12-3.

13. Linn L, Yager J, Leake B. Physicians' attitudes towards the legalization of marijuana use. West $J$ Med. 1989;150:714-7.

14. Kilmer JR, Hunt SB, Lee CM, Neighbors C. Marijuana use, risk perception, and consequences: is perceived risk congruent with reality? Addict Behav. 2007;32(12):3026-33.

15. Agostinelli G, Miller WR. Drinking and thinking: how does personal drinking affect judgments of prevalence and risk? J Stud Alcohol. 1994;55(3):327-37.

16. Sherman DA, Nelson LD, Steele CM. Do messages about health risks threaten the self? Increasing the acceptance of threatening health messages via self-affirmation. Personal Soc Psychol Bull. 2000;26(9):1046-58.

17. Clarke VA, Lovegrove H, Williams A, Machperson M. Unrealistic optimism and the health belief model. J Behav Med. 2000;23(4):367-76.

18. Cohen JJ, Gabriel BA, Terrell C. The case for diversity in the health care workforce. Health Aff. 2002;21(5):90-102.

19. Flanigan TS, McFarlane E, Cook S, eds. Conducting survey research among physicians and other medical professionals: a review of current literature. Proceedings of the Survey Research Methods Section, American Statistical Association; 2008.

20. Baldwin DC, Hughes PH, Conrad SE, Storr CL, Sheehan DV. Substance use among senior medical students: a survey of 23 medical schools. JAMA. 1991;265:2074-8. 